\title{
Potential role of 3DUS in infants and children
}

\author{
Michael Riccabona
}

Received: 9 December 2010 / Accepted: 3 January 2011

(C) Springer-Verlag 2011

\begin{abstract}
The objective of this review is to discuss basic technical aspects as well as potential pediatric applications of three-dimensional ultrasound (3DUS). Different 3DUStechniques are already commercially available. Most commonly, a transducer-integrated motor drives a scan-head to acquire a volumetric dataset, which is then reconstructed and viewed using various post-processing techniques. It has been proved feasible to apply 3DUS with pediatric transducers in typical pediatric investigations. Based on our own experiences and on review of the literature, 3DUS can be successfully used in infants and children for brain, spine, cardiac, urinary tract (particularly assessment of hydronephrosis, and virtual cystoscopy), and female genital (e.g., for assessment of uterine malformations) US, and for various other applications, particularly in small parts. Power Doppler data can be integrated, allowing 3DUSangiography. Thus, 3DUS promises to become a useful
\end{abstract}

Disclaimer The supplement this article is part of is not sponsored by the industry. The author is a member of the European Advisory Board on 3DUS of Kretz, and as such his department has received research support (equipment loans) several times, and the author has been an invited lecturer and trainer for the company.

\author{
M. Riccabona \\ Department of Radiology, Division of Pediatric Radiology, \\ Medical University, Universitätsklinikum LKH, \\ Graz, Austria \\ M. Riccabona $(\square)$ \\ Department of Radiology, Division of Pediatric Radiology, \\ LKH Graz, University Hospital, \\ Auenbruggenplatz, \\ 8036 Graz, Austria \\ e-mail: michael.riccabona@medunigraz.at
}

adjunct for imaging children, particularly as it enhances ultrasound by offering additional, previously inaccessible planes, rendering options and surface assessments. 3DUS should be increasingly exploited, thus hopefully helping reduce the need for more invasive or burdening (e.g., ionizing radiation) investigations in children.

Keywords Neonates - Infants and children · Three-dimensional ultrasound (3DUS) · Neurosonography Kidney $\cdot$ Urogenital tract · Cystoscopy

\section{Introduction}

US is the mainstay of pediatric radiology and not only due to radiation protection issues. As children offer superb conditions for performing US examinations with highfrequency high-resolution transducers utilizing the partially non-ossified access in children, many clinical questions can be reliably answered sonographically, where in adults there would often be need for other cross-sectional imaging. Any technique that promises to improve US and to increase its diagnostic yield should be welcomed and exploited.

Three-dimensional US is a relatively new technique that was introduced around 1995. It was initially used for gynecologic and obstetric imaging; other applications in adults followed [1-5]. With the introduction of transducers that are useful in children, the first efforts have been undertaken to evaluate its potential in pediatric imaging [612]. This review will (1) present basic methodical aspects of 3DUS to enable an understanding of the underlying physics with its implications for scan technique and results, (2) present some of our local experience, and (3) review the literature. 


\section{Technique}

There are several techniques available:

- Freehand-scanning with an assumption-based algorithm for reconstruction of volumetric data

- Freehand-scanning using a position sensor at the transducer, which allows defining the position in space of each individual 2DUS image and thus allowing an accurate 3D reconstruction [13]

- Dedicated 3DUS transducers, which usually have a motor integrated into the scan-head that performs a sweep of a 2DUS-array over a predefined region of interest for acquiring the volumetric data

- And, the newest technology, dedicated volume-matrix transducers using multiple crystals that form a 2Dmatrix in the scan-head and allow for simultaneous acquisition of a volume

Whichever method is used, the data sets are then postprocessed similar to data from $\mathrm{CT}$ and MRI, with various presentation modes, such as surface rendering, maximum- and minimum-intensity projections, and volume rendering $[1,2]$. Interactive navigation using multi-planar reformatting has proved particularly beneficial for differential diagnostic purposes and for assessment of complex anatomical abnormalities. Rendering tools are most useful for conspicuously visualizing surfaces or tortuous structures.

There are, however, several limitations. First, acquisitions may be distorted by motion artifact (caused by any motion occurring during the acquisition). Second, shadowing will transfer into the 3D dataset and thus represent a potential source of error. Third, restricted access will always restrict the potential of 3DUS (since only actually acquired data can be processed) [14]. Fourth, no directional color Doppler or flow information can be integrated into the 3DUS dataset. Fifth, usually the resolution of reconstructed planes is inferior to the resolution of native two-dimensional US (i.e., the image in the acquisition plane). These and other artifacts have to be considered when reading 3DUS to avoid misinterpretation.

\section{Potential in typical pediatric applications}

3D neurosonography

Using the transfontanellar access and a dedicated pediatric 3DUS transducer one can easily acquire a volumetric dataset of the neonatal brain. Reviewing is performed mostly in orthogonal planes (Fig. 1); in particular, the reconstructed axial plane is valuable for enhancing anatomic analysis and comparison with other cross-sectional imaging, such as CT and MRI (Fig. 2). The ability of 3DUS to show any particular structure in all three orthogonal planes allows an improved definition of individual structures and may prove valuable for differential diagnoses, both for fetal and neonatal applications (Fig. 3) [9, 10, 12, 15]. The ventricles can be shown nicely; when enlarged, applying 3D-rendering techniques with grey-scale volumerendering 3DUS inversion, an intuitive visualization of the

Fig. 1 Transfontanellar brain three-dimensional ultrasound (3DUS). Standard display of a 3DUS volume of a normal neonatal brain, using the three standard orthogonal planes (sagittal, coronal, axial) as well as a compound view (lower right) that correlates the planes to improve orientation and navigation
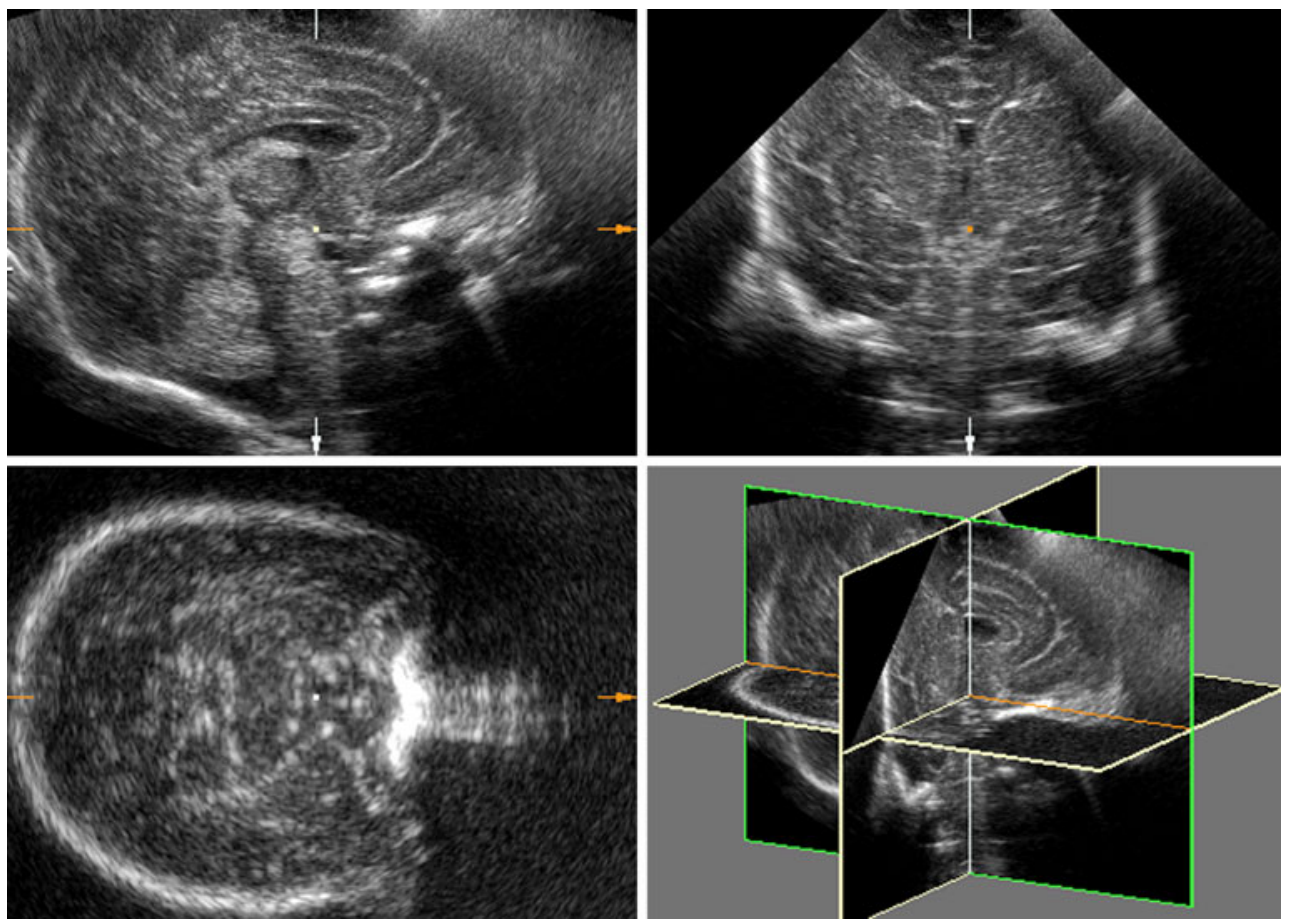


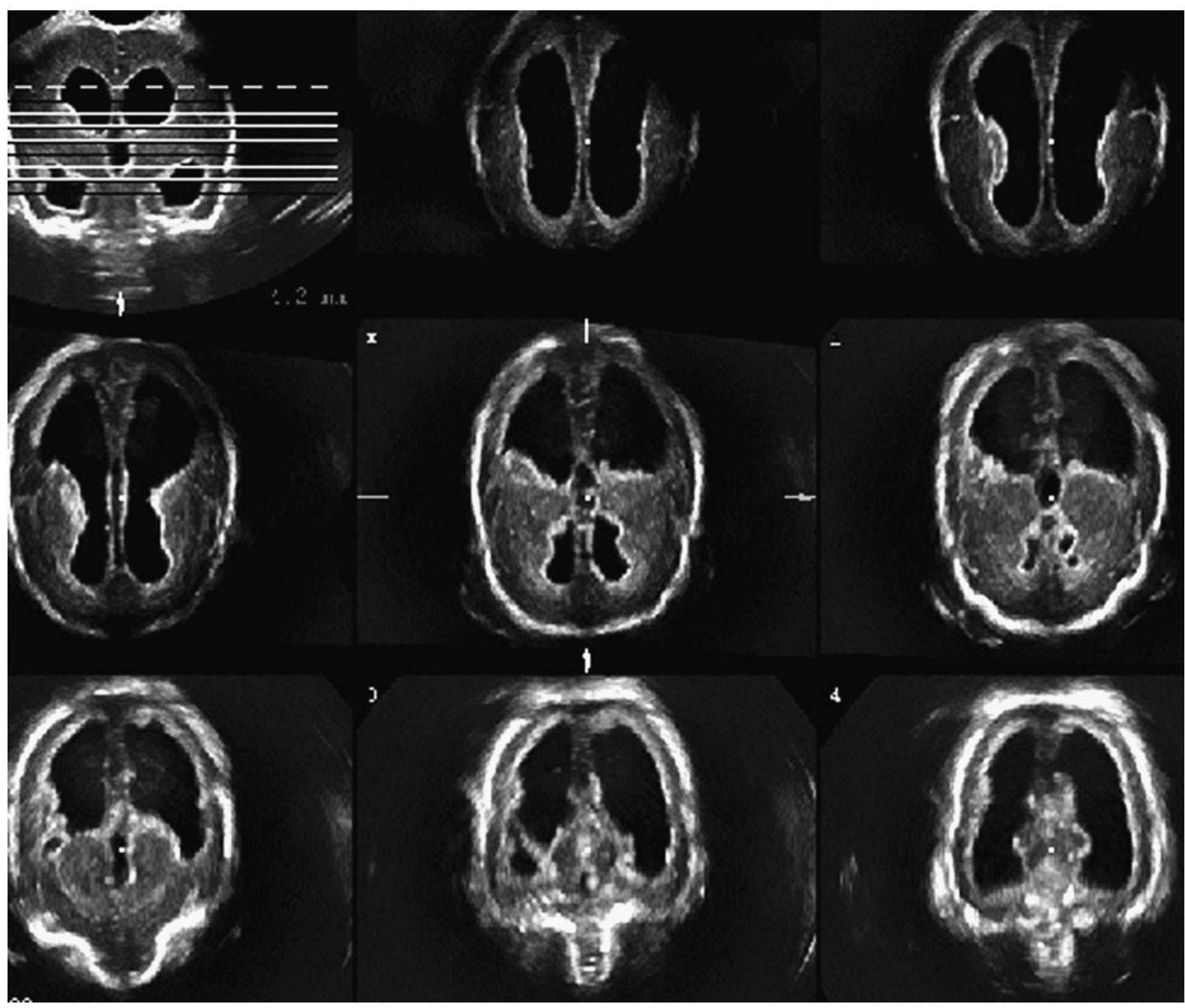

Fig. 2 Axial three-dimensional ultrasound (3DUS) views ("tomographic US imaging") in hydrocepehalus. Serial axial thick-slab reconstructions of a transfontanellar brain 3DUS in a baby with hydrocephalus,

dilated ventricles, and even (semi-)automated ventricular volume calculations, offers new options, not only in terms of diagnosis and follow-up, but also for discussing findings with physicians and parents (Fig. 4) [7, 9, 16, 17]. A new option is the use of surface rendering for visualization of the brain surface. This tool, using curved volume rendering settings, may widen the scope of US to include assessment of skull injury, and may improve the ability of US to detect gyration disorders (Fig. 5). Thick-slab rendering may enhance subtle findings, such as in periventricular leucomalacia. Integrating color Doppler data into the 3DUS dataset allows conspicuous visualization of several vessels. In particular, abnormal tortuous vessels, such as in arteriovenous malformations, can convincingly be displayed, along with their feeding arteries and major draining vessels displaying pathology in a standardized axial orientation, thus easily comparable to CT and MRI

(Fig. 6) [9, 18]. As the volume acquisition is quick and most of the imaging analysis is performed at the workstation or the US device, without having to be at the patient's bedside. This technique reduces the burden, especially on very sick neonates or preterm infants, by shortening the scan time [10]. However, a conventional two-dimensional US scan for first orientation, and also for adapting image parameters, remains essential. High-resolution US of the brain surface cannot be replaced in 3DUS due to restricted 3DUS resolution, particularly in reconstructed planes. Directional color Doppler and flow evaluation must still be performed by two-dimensional US [19].

Other promising 3DUS applications in pediatric neurosonography include visualization of the orbits, and, as long as a non-ossified access persists, the spinal canal; the 
Fig. 3 Multi-planar display in neonatal 3D neurosonography. Brain three-dimensional ultrasound (3DUS) in a preterm baby with suspected intraventricular hemorrhage displayed in three orthogonal planes. The reconstructed axial plane (lower left) demonstrates that the bleeding is confined to the choroid plexus, and that there is no clot in the ventricle. Orientation is facilitated by a white dot that marks the corresponding point in all the three planes
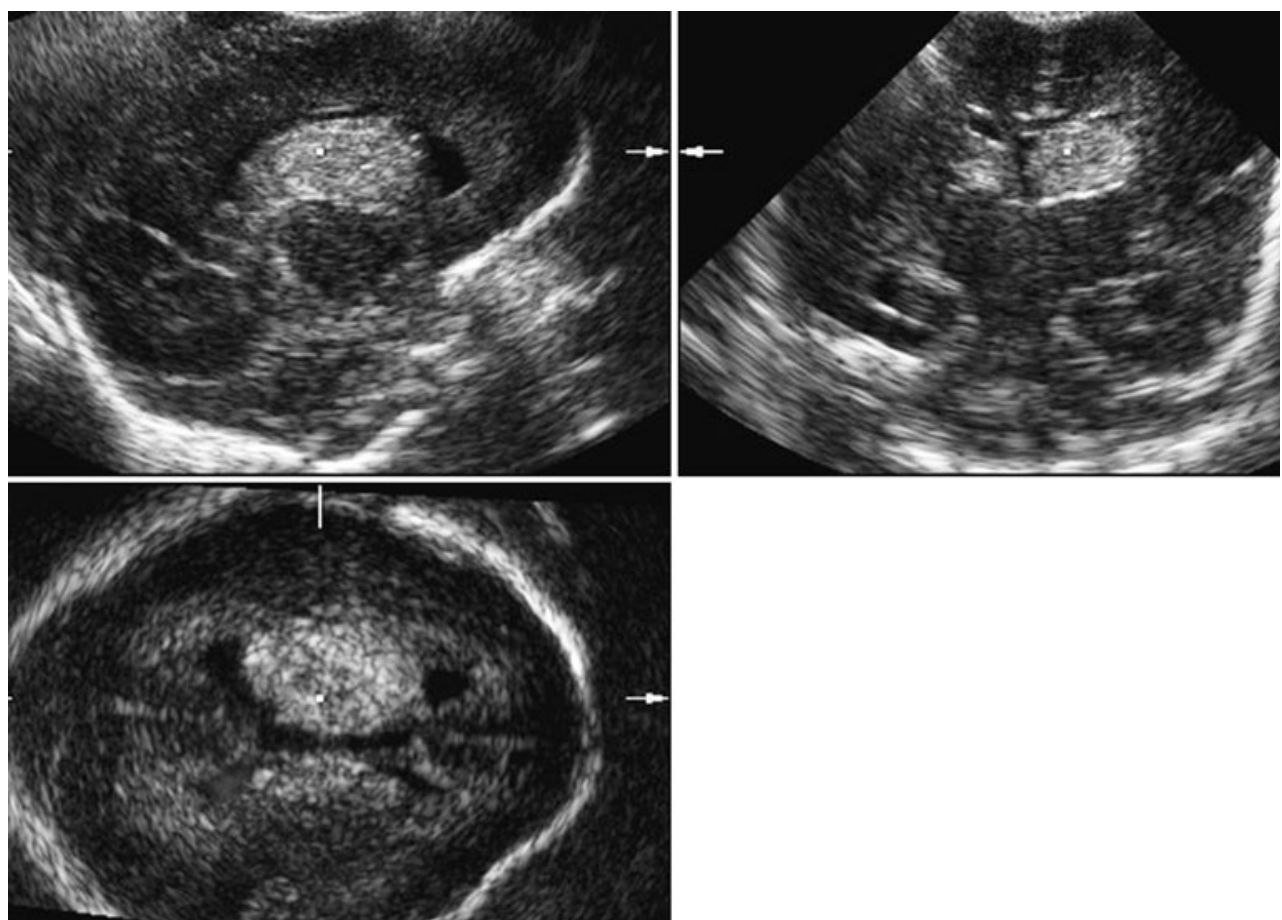

applicability has been shown, but no thorough research exists into its clinical value [17].

\section{D urogenital sonography}

In the pediatric urinary tract, 3DUS has been applied for evaluating the kidneys and the urinary bladder. In the kidney, the superior potential of 3DUS for volume

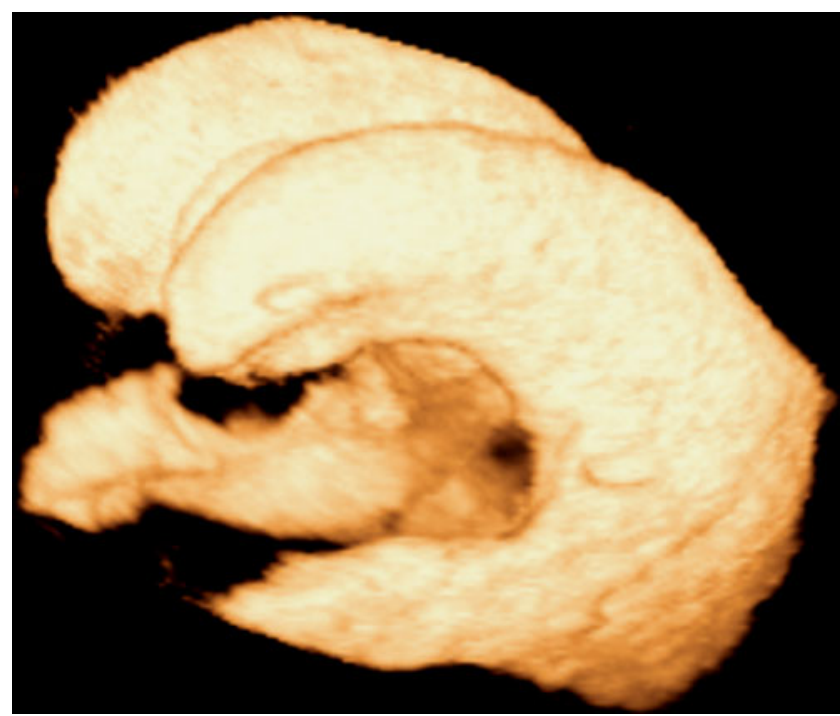

Fig. 4 Ventricular rendering of a three-dimensional ultrasound (3DUS) acquisition in neonatal hydrocephalus. The rendered view using a greyscale inversion volume rendering technique conspicuously displays the dilated lateral ventricles. This segmented 3DUS dataset can also be used for (semi-)automated ventricular volume calculation assessment of structures with more complicated shape has been utilized to accurately calculate renal parenchymal volume, which is most helpful in hydronephrotic kidneys $[8,17,20,21]$. This is achieved by subtraction of the segmented dilated collecting system from the overall kidney volume (Fig. 7). When performed in both kidneys, split renal parenchymal volume can be calculated, which has been found comparable to results from DMSA scintigraphy and MR urography [22]. As renal parenchymal

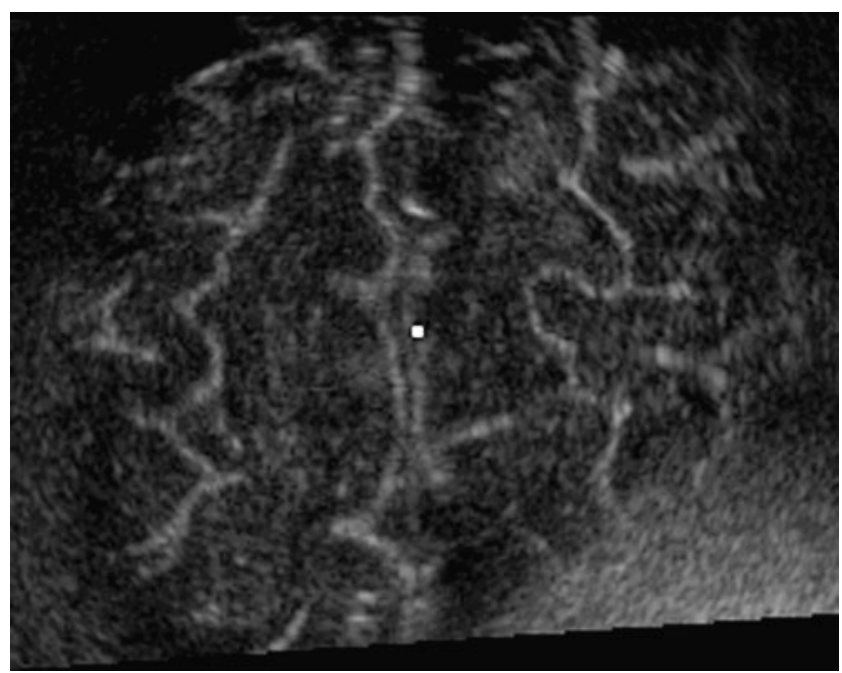

Fig. 5 Brain surface display using three-dimensional ultrasound (3DUS). This view of the brain surface in a slightly premature baby, showing the paramedian gyri, is reconstructed from the same dataset as Fig. 1 using a curved surface rendering algorithm. The white dot marks the interhemispheric fissure 
Fig. 6 Power Doppler threedimensional ultrasound (3DUS) in neonatal cerebral arteriovenous (vein of Galen) malformation. 3D-power Doppler images demonstrate the vasculature in three orthogonal planes; additionally, the rendered view provides a conspicuous "angiographic" image (bottom right)

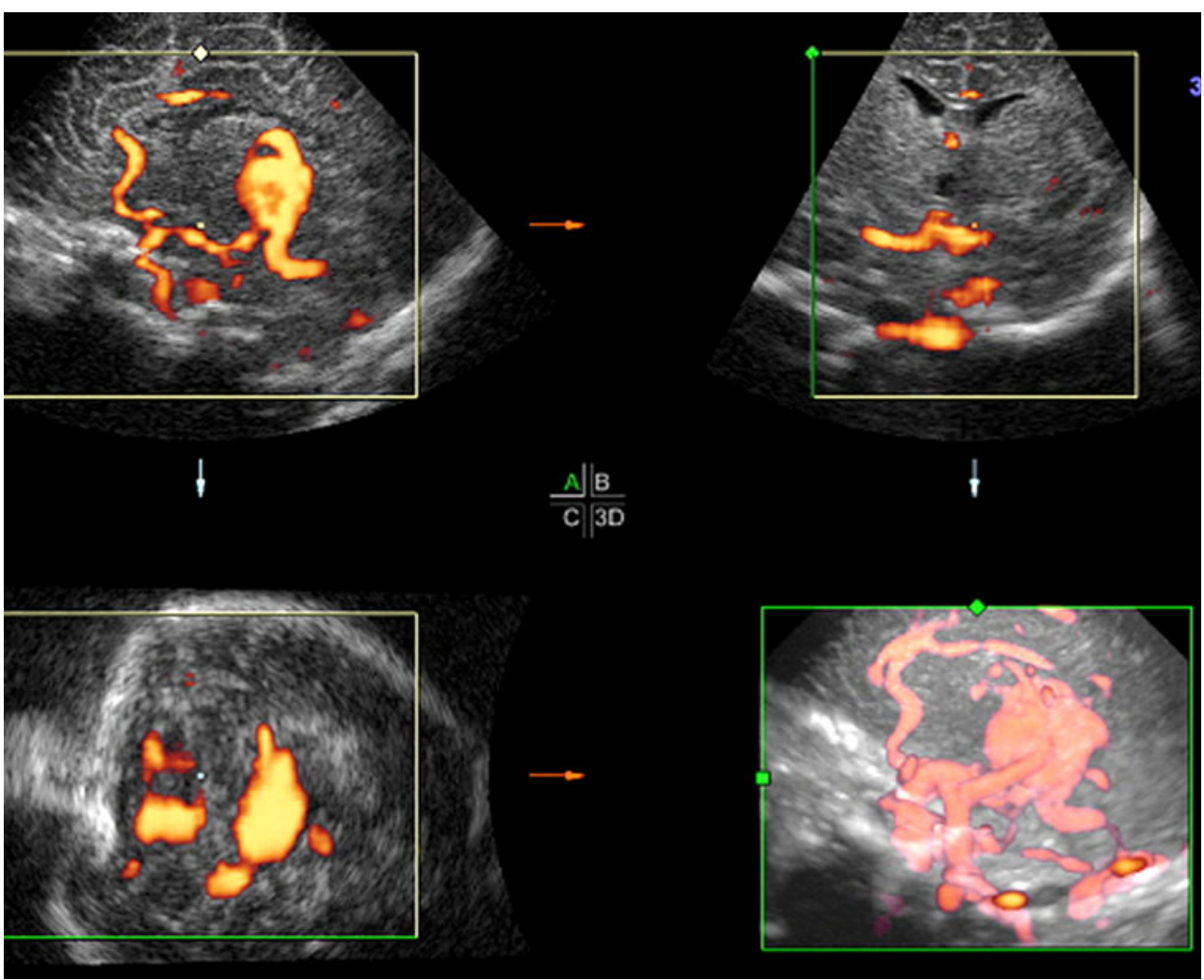

volume growth rate and changes in split renal parenchymal volume are critical factors for treatment decisions in patients with significant hydronephrosis, such as in uretero-pelvic junction obstruction, additional 3DUS can improve the usefulness of US in follow-up and thus may reduce the need for scintigraphy. The volume calculations can be performed either by manual outlining of the outer contour of the measured structure or by (semi-)automated volume calculation software as provided by the various vendors. For delineation, segmentation and volume calcu-
Fig. 7 Three-dimensional ultrasound (3DUS) in hydronephrosis: kidney volume calculation. Using manual outlining or greyscale inversion segmentation to define the dilated pelvi-caliceal system (bottom right) allows subtraction of this sub volume from the outlined entire renal volume for accurate calculation of renal parenchymal volume in hydronephrotic kidneys

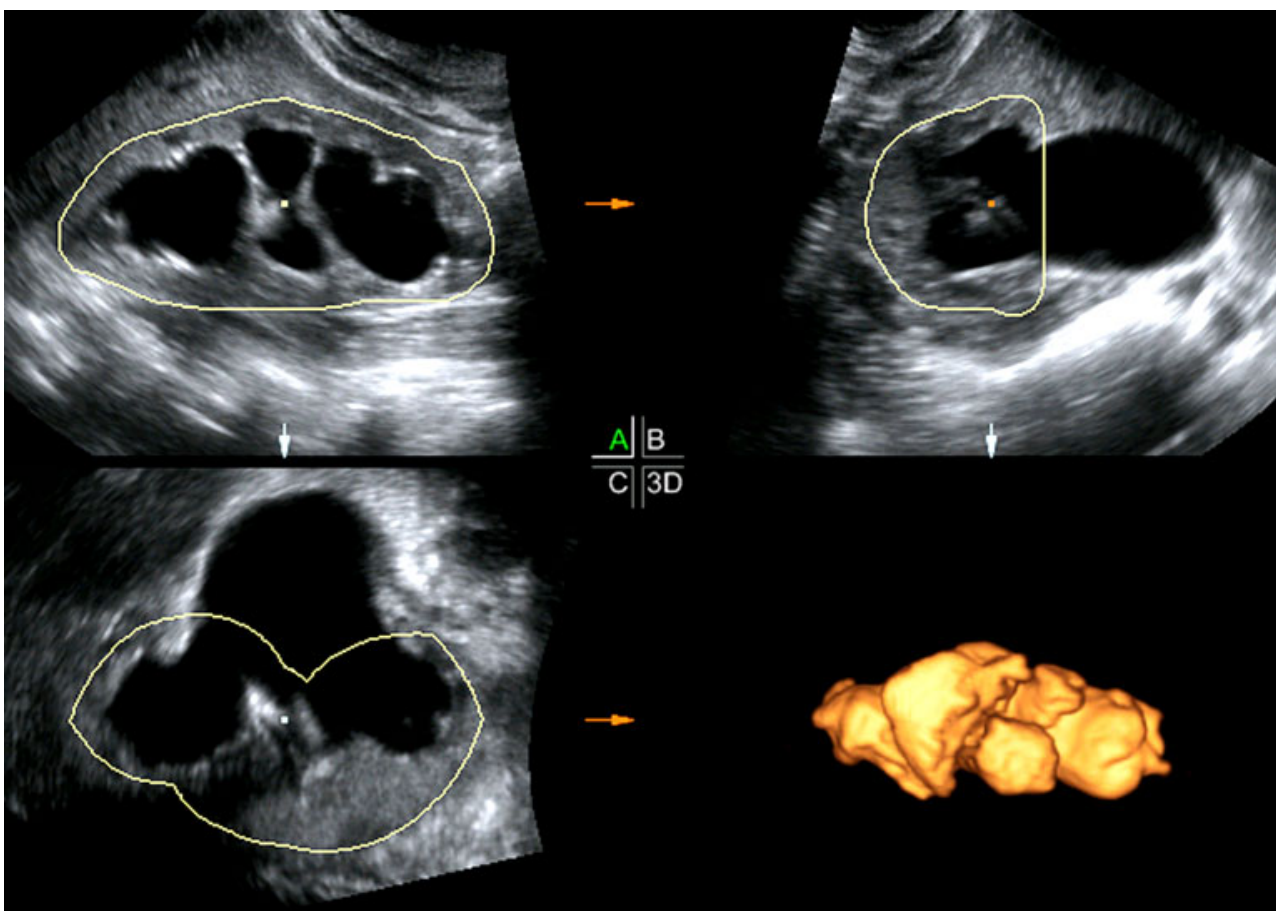


lation of a dilated collecting system, grey-scale inversion with volume rendering can help speed up the process. This rendered inverted view of the dilated system is also helpful for visualization of the pelvicalyceal-ureter unit, which aids in differentiating non-obstructed dilatation in malformed calices, such as megacalicosis, from the typical ballooned shape of an obstructive dilated system. It can also be helpful for assessment of caliceal diverticuli and tertiary calices $[8,17]$. The same principle of volume assessment may apply to tumor volume calculation, which is potentially beneficial for following patients with renal or bladder tumors during chemotherapy (Fig. 8).

If amplitude-coded color Doppler data are included in the 3DUS dataset, a conspicuous visualization of additional renal arteries and their relation to the collecting system becomes feasible. Superior demonstration of topographical vascular distribution can also be achieved, which is useful for assessment of inflammation and scarring (Fig. 9). One caveat is that very small vessels and subtle vascular abnormality may be missed due to the limited resolution, particularly of power Doppler 3DUS.

In the urinary bladder, 3DUS was initially used for more accurate calculations of volume in irregularly shaped bladders. This is particularly helpful for assessment of residual urine or content of a neurogenic bladder where, due to the atypical bladder shape, the conventional US estimates may be very inaccurate [23]. Automated bladder volume-scanners have been marketed; however, although these work well with normal bladder shapes, they may be prone to significant error in irregularly shaped bladders and other unusual situations, as one cannot visualize the bladder and thus cannot correct for mistakes. These devices should only be used when the bladder shape and the anatomical situation in the lower pelvis has previously been investigated by a comprehensive conventional US study.

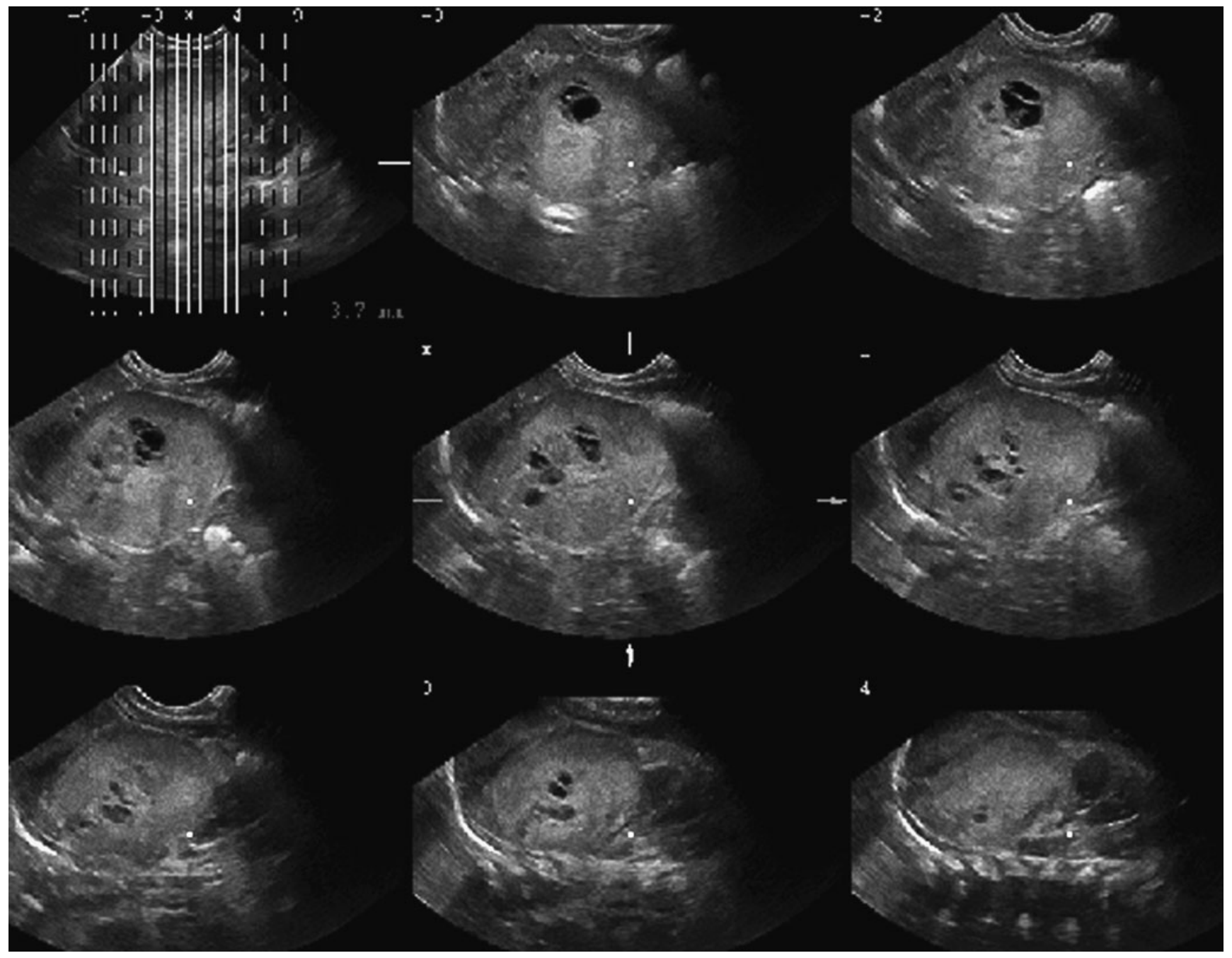

Fig. 8 Three-dimensional ultrasound (3DUS) in renal tumor. This tomographic multi-sectional display of a renal tumor demonstrates the ability to visualize the entire tumor, similar to CT and MR. From these serial consecutive images an accurate tumor volume-calculation can be performed 


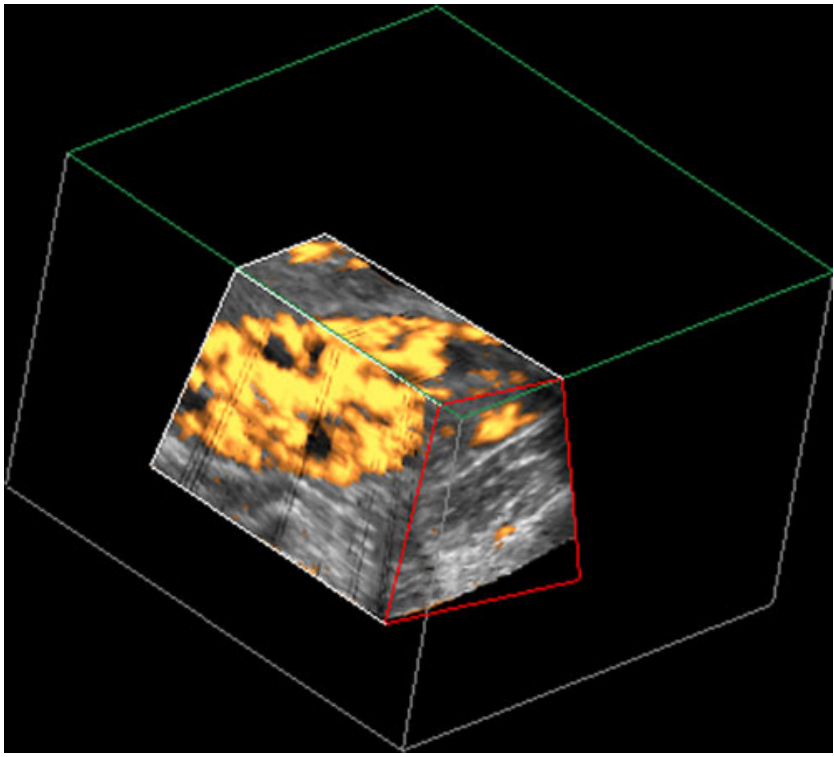

Fig. 9 Renal power Doppler three-dimensional ultrasound (3DUS) in acute pyelonephritis. The 3DUS box-image demonstrates a perfusion defect in the lower pole of the kidney in a child with acute pyelonephritis

A newer 3DUS application is virtual cystoscopy. This is based on surface rendering with a view-point inside the bladder. It renders images of the internal bladder wall, allowing focusxed visualization of important areas, such as the trigone with the bladder neck and the ureteric ostia, or of diverticuli [24, 25] (Fig. 10). Real-time 3DUS (so-called 4DUS) can be performed with modern equipment and does not only reveal anatomic, but also functional information (for example, intermittent gapping of an ostium during vesico-ureteric reflux, or intermittent posing of an ureterocele during the maximum of the ureteric peristaltic wave). Virtual cystoscopy may prove helpful for reducing the need for endoscopic cystoscopy in children, as usually no biopsies are necessary and only anatomic or functional information are required clinically [25]. At present, the spatial resolution of 3D- and 4DUS is sufficient to visualize all major structures in infants and children older than 1 year. One might speculate that with this new virtual cystoscopy information, 3DUS may help improve indications for performing voiding cysto-urethrography through refined selection of those children with high suspicion for significant and treatmentrelevant vesico-ureteral reflux, as these have lateralized and gapping ostia that can be detected by 3DUS. Other infants and children with less complicated vesico-ureteral reflux, which will probably maturate without (surgical) treatment, mostly have normal ostial shapes and positions, which can be identified by virtual cystoscopy; thus, indication for voiding cysto-urethrography can be postponed in these patients, potentially completely avoided, and would save an invasive procedure as well as exposure to ionizing radiation.
Fig. 10 Virtual cystoscopy based on a three-dimensional ultrasound (3DUS) dataset. 3DUS-based virtual cystoscopy, i.e. rendering of the internal surface of the urinary bladder, demonstrates a large ureterocele (arrow) that herniates into the bladder neck and causes bladder outlet obstruction. The secondary trabeculation of the enlarged bladder is easily appreciated. A bladder catheter (arrowhead) is in place to ensure urinary drainage
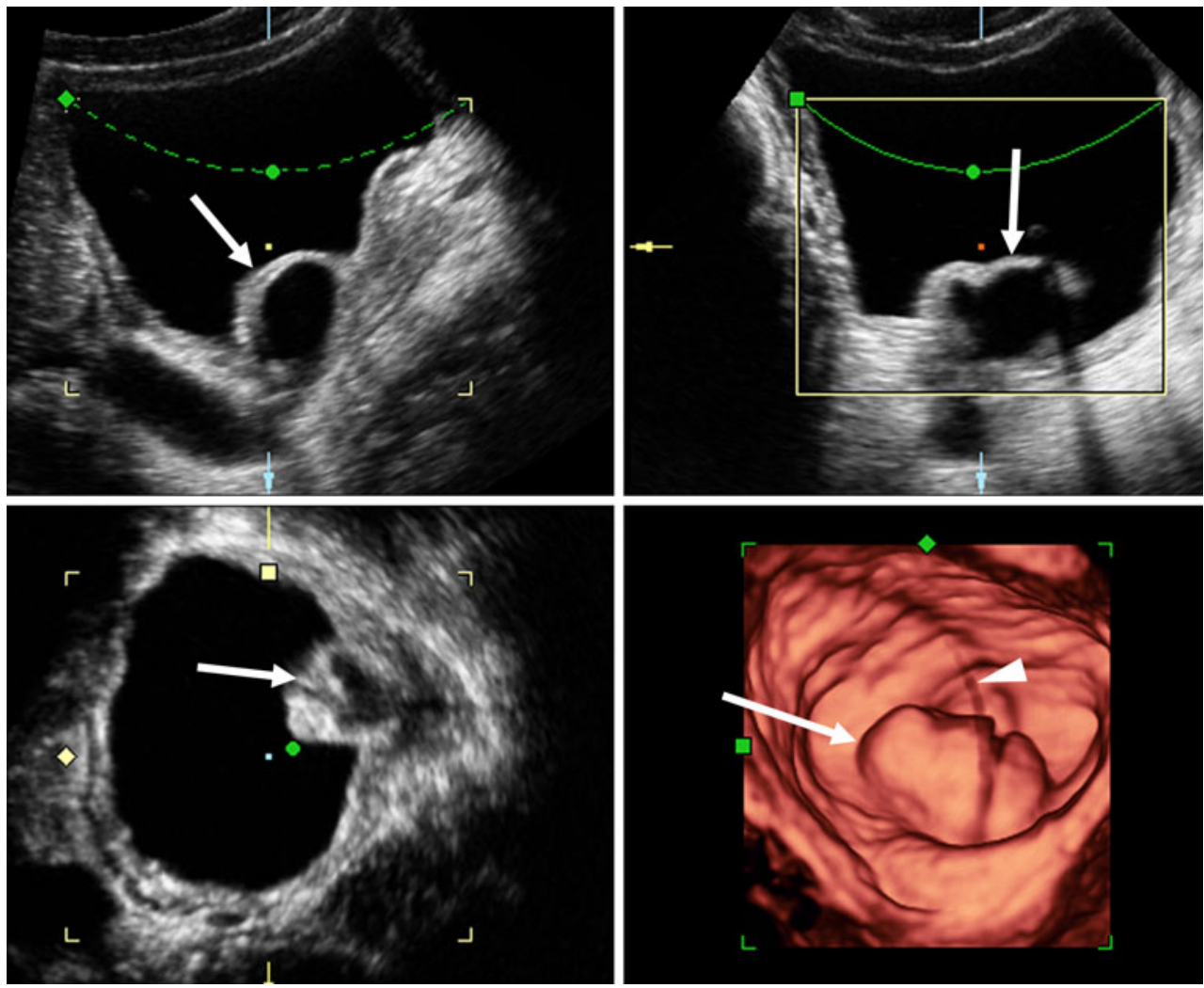
Other potential pediatric 3DUS applications

There are several potentially useful 3DUS indications. As in adults, 3DUS can be helpful for assessment of malformations of the internal female genitalia, e.g., in uterine duplications (Fig. 11) [1, 2, 8, 26]. Additionally, pelvic floor assessment appears feasible using a perineal approach. There are also reports of using a thin endorectal 3DUS probe for assessment of pelvic floor anatomy and pathology. However, the clinical potential of these incidental observations and case reports has not been validated [27].

Other small-part applications, besides improved volume calculations, are investigation of musculoskeletal structures, and skin and soft-tissue lesions, where 3DUS may be valuable are in conditions where an additional reconstructed plane that is not accessible by conventional US offers crucial information, or for very torturously shaped abnormalities $[1,2,8,17$, 28-30]. This may prove helpful in fractures that sometimes can be difficult to see on plain film, or for abscesses with fistula formation $[8,17,31]$.

Naturally, the scrotum, lymph nodes, the thyroid gland, subcutaneous cysts or tumors, and similar structures can be imaged by 3DUS; however, except for improved volume calculation of complex-shaped organs and lesions, there has yet been little proven benefit from these applications. The same applies to 3DUS of the neonatal hip. It is feasible, particularly if the Graf method is used, but assessment at

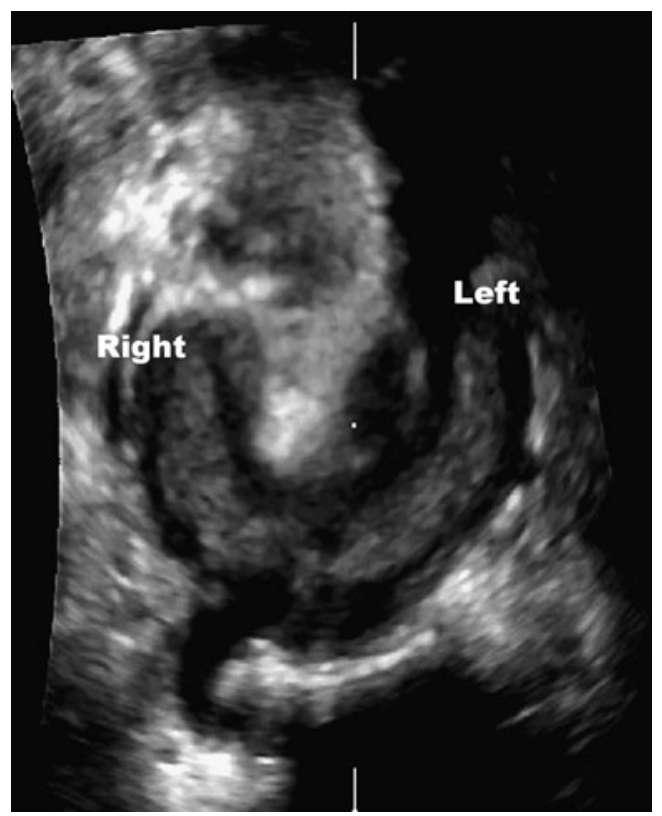

Fig. 11 Three-dimensional ultrasound (3DUS) of uterine duplication in a 13-year-old girl. This coronal thick-slab reconstruction of a duplicate uterus convincingly displays the two uterine horns with their two central echogenic endometrial cavities. As this scan-plane is unavailable in conventional transabdominal US, 3DUS significantly improves the potential of US in the diagnosis classification of uterine malformations in children. This application is well-known in adult sonography the console or workstation takes much longer than the conventional US investigation, and therefore does not seem beneficial since dynamic assessment would have to be added regardless.

3DUS can be performed in other abdominal organs; various applications have been found useful in adults but are less feasible or less commonly used in pediatrics. The only current useful 3DUS application in the pediatric abdomen is volume calculation of structures that do not match geometrical shapes, in which no reliable equations can be applied to estimate a proper volume from conventional US [17, 32, 33]. In adults, 3DUS has been found helpful for performing transjugular intra-hepatic porto-systemic shunt-procedures, punctures and biopsies. As these are far less frequently performed in children, such applications have not been assessed for pediatric use, however, may prove beneficial in future.

Color Doppler 3DUS may be helpful for enhanced visualization and presentation of complex vascular anatomy and abnormality, e.g., to assess the extent of a vascular malformation. This potential application is challenged by the limited resolution of color Doppler in 3DUS and by the limited range of acquisition, which will often hinder the visualization of the entire lesion.

Finally 3D- and 4DUS can be applied to the pediatric heart and large vessels, particularly in neonates, who offer an ideal US access by the persisting thymus and the non-ossified parts of the sternum and ribs. Here, similarly to fetal US, fast acquisitions using either gating techniques (e.g. spatialtemporal image correction or correlation technology in the Kretz-systems; Kretztechnik, Zipf, Austria) or real-time matrix volume-transducers (as offered in, e.g., Philips iU22, Philips Medical Systems, Best, The Netherlands) improve visualization of the internal cardiac structures, and enable visualization and observation of valves and their function and dysfunction $[34,35]$. By providing a volumetric dataset throughout diastole and systole 4DUS has the potential to improve volume-based calculations such as left and right ventricular volume, ejection fraction, or stroke volume. This might improve US accuracy and functional assessment, particularly with complex pathological anatomy, where conventional US estimates are less accurate. These patients are currently examined by MRI or catheter angiography. In future, integration of directional Doppler data may enhance the potential of 4DUS. Currently, unidirectional color-data can be integrated with grey-scale images for visualization of shunts and insufficiencies as well as of the anatomy of the large vessels; however, this does not allow flow quantification.

\section{Discussion}

3DUS has been shown clinically useful in a number of applications in adults. In obstetrics in gynecology it has 
become an established routine tool in the diagnostic workup of various conditions $[1-5,15,18,20,21,26-33]$.

However, in children, 3DUS is still not widely used, although initial experiences on its feasibility and clinical potential have been reported $[6-10,12,16,17,19,22,23$, $25,36]$. This may be due to restricted access to 3DUS units and transducers, or due to problems with motion artifacts when scanning un-cooperative children. There is undoubtedly a great potential for improving diagnostic US in children by using 3DUS as outlined above. To optimally exploit this noninvasive diagnostic tool, pediatric radiologists should be encouraged to engage with 3DUS and study its potential benefit in various applications. After an initial learning curve, practical scanning is not so difficult if the operator is used to handling children. High-quality acquisitions are often easily achievable even in small children without the need for sedation or restraint. Further research is needed to thoroughly assess its diagnostic role and clinical efficacy.

Some restrictions of 3DUS have to be acknowledged, such as scanning children with large transducers; restricted resolution, particularly in reconstructed planes; the possibility of motion artifacts; the time necessary for post-processing; and additionally several intrinsic artifacts [14]. However, these setbacks are partially outweighed by the potential diagnostic benefit and a shorter scan [10, 19]. If significant additional diagnostic information can be retrieved from 3DUS, the additional time necessary for post-processing and datahandling at the workstation may be acceptable, as it currently is for CT and MRI.

\section{Conclusion}

3DUS has great potential for improving and widening US diagnosis in the pediatric brain, abdomen, urogenital tract, and various small parts. Additionally, 4DUS has become feasible even in infants and children with the promise to improve the potential of US in assessing functional phenomena, such as in virtual cystoscopy or in 4D cardiac US. More adequate transducers and devices should, and hopefully will be made available for the pediatric US community, enabling the pediatric radiologist to integrate this promising tool in routine practice. As for several other modalities in pediatric radiology, more in-depth research on its diagnostic potential and efficacy is still needed.

\section{References}

1. Merz E (ed) (1998) 3-D ultrasound in obstetrics and gynecology. Lippincott, Williams \& Wilkins, Philadelphia

2. Nelson TR, Pretorius DH, Fenster A et al (1999) Three-dimensional ultrasound. Lippincott, Williams \& Wilkins, Philadelphia
3. Pretorius DH, Nelson TR (1995) Fetal face visualization using three dimensional ultrasound. J Ultrasound Med 14:349-356

4. Pretorius DH, Nelson TR (1998) Three-dimensional ultrasound in gynecology and obstetrics: a review. Ultrasound Q 14:218-233

5. Weinraub Z, Maymon R, Shulman A et al (1996) Threedimensional saline contrast hystero-sonography and surface rendering of uterine cavity pathology. Ultrasound Obstet Gynecol $8: 277-282$

6. Gilmore JH, Gerig G, Specter B et al (2001) Infant cerebral ventricle volume: a comparison of $3 \mathrm{D}$ ultrasound and magnetic resonance imaging. Ultrasound Med Biol 27:1143-1146

7. Nagdyman N, Walka MM, Kampmann W et al (1999) 3D ultrasound quantification of neonatal cerebral ventricles in different head positions. Ultrasound Med Biol 25:895-900

8. Riccabona M, Fritz G, Ring E (2003) Potential applications of three-dimensional ultrasound in the pediatric urinary tract: pictorial demonstration based on preliminary results. Eur Radiol 13:2680-2687

9. Riccabona M, Nelson TR, Resch B et al (2003) Potential of threedimensional ultrasound in neonatal and pediatric neurosonography: a pictorial essay. Eur Radiol 13:2082-2093

10. Salerno CC, Pretorius DH, SvW H et al (2000) Three-dimensional ultrasonographic imaging of the neonatal brain in high risk neonates: preliminary study. J Ultrasound Med 19:549-555

11. Seward JB, Belohlavek M, O'Leary PW et al (1995) Congenital heart disease: wide-field, three-dimensional, and four-dimensional ultrasound imaging. Am J Card Imaging 9:38-43

12. Stanojevic H, Kurjak A (2002) Three-dimensional (3D) ultrasound-a useful imaging technique in the assessment of neonatal brain. Perinat Med 30:74-83

13. Matre K, Stokke EM, Martens D et al (1999) In vitro estimation of kidneys using three-dimensional ultrasonography and a position sensor. Eur J Ultrasound 10:65-73

14. Nelson TR, Pretorius DH, Hull AD et al (2000) Sources and impact of artifacts on clinical 3DUS imaging. Ultrasound Obstet Gynecol 15:1-11

15. Monteagudo A, Timor-Tritsch IE, Mayberry P (2000) Threedimensional transvaginal neuro-sonography of the fetal brain: 'navigating' in the volume scan. Ultrasound Obstet Gynecol 16:307-313

16. Csutak R, Unterassinger L, Cl R et al (2003) Three-dimensional volume measurement of lateral ventricles in preterm and term infants: evaluation of a standardised computer-assisted method in vivo. Pediatr Radiol 33:104-109

17. Riccabona M (2005) Pediatric three-dimensional ultrasound: basics and potential clinical value. Clin Imaging 29:1-5

18. Lyden PD, Nelson TR (1997) Visualization of cerebral circulation using three-dimensional power Doppler ultrasound imaging. $\mathrm{J}$ Neuroimaging 7:35-39

19. Fritz GA, Riccabona M, Weitzer C et al (2005) Dreidimensionaler Ultraschall (3DUS) des neonatalen Gehirns: klinische Anwendbarkeit bei intensivgepflegten Neugeborenen. Ultraschall Med 26:209-306

20. Riccabona M, Nelson TR, Pretorius DH et al (1995) Distance and volume measurements using three dimensional ultrasound. J Ultrasound Med 14:881-886

21. Treece G, Prager R, Gee A et al (2001) 3D ultrasound measurement of large organ volume. Med Image Anal 5:41-54

22. Riccabona M, Fritz GA, Schoellnast H et al (2005) Hydronephrotic kidney: pediatric three-dimensional US for relative renal size assessment-initial experience. Radiology 236:276-283

23. Riccabona M, Nelson TR, Pretorius DH et al (1996) In vivo threedimensional sonographic measurement of organ volume: validation in the urinary bladder. J Ultrasound Med 15:627-632

24. Dahia N (2006) Virtual cystoscopy using 3D ultrasound in evaluation of urinary bladder lesions. J Ultrasound Med 25:S82 
25. Riccabona M, Pilhatsch A, Haberlik A et al (2008) Threedimensional ultrasound based virtual cystoscopy of the pediatric urinary bladder: a preliminary report on feasibility and potential value. JUM 27:1453-1459

26. Jurkovic D, Geipel A, Gruboeck K et al (1995) Three-dimensional ultrasound for the assessment of uterine anatomy and detection of congenital anomalies: a comparison with hysterosalpingography and two-dimensional sonography. Ultrasound Obstet Gynecol 5:233-237

27. Valsky DV, Yagel S (2007) Three-dimensional transperineal ultrasonography of the pelvic floor. JUM 26:1373-1387

28. Hashimoto S, Goto H, Hirooka Y et al (1999) An evaluation of three-dimensional ultrasonography for the measurement of gallbladder volume. Am J Gastroenterol 94:3492-3496

29. Mende U, Krempien R, Hassfeld S et al (2002) 3D-ultrasound: a valuable adjunct for therapy planning and follow-up of head and neck tumours. Ultraschall Med 23:101-107

30. Schlogl S, Werner E, Lassmann M et al (2001) The use of threedimensional ultrasound for thyroid volumetry. Thyroid 11:569-574
31. Hünerbein M, Raschke M, Haas NP et al (2000) Threedimensional ultrasonography: new prospects for ultrasound imaging of bone. Lancet 355:116-117

32. De Odorico I, Spaulding KA, Pretorius DH et al (1999) Normal splenic volumes estimated using three-dimensional ultrasonography. J Ultrasound Med 18:231-236

33. Lang H, Wolf GK, Prokop M et al (1999) Three-dimensional ultrasound for volume measurement of liver tumors. Chirurg 70:246-250

34. Marx GR, Sherwood MC (2002) Three-dimensional echocardiography in congenital heart disease: a continuum of unfilled promises? No. A presently clinically applicable technology with an important future? Yes. Pediatr Cardiol 23:266-285

35. Nelson TR, Pretorius DH, Sklansky M et al (1996) Three-dimensional echocardiographic evaluation of fetal heart anatomy and function: acquisition, analysis and display. J Ultrasound Med 15:1-9

36. Fritz GA, Riccabona M, Bohdal G et al (2003) Accuracy of renal volume assessment in children by three-dimensional sonography. Rofo Fortschr Geb Rontgenstr Neuen Bildgeb Verfahr 175:540-546 